\title{
Empowerment durch Kapazitätsentwicklung im Quartier - erste Ergebnisse und Einschätzung eines Erhebungsinstruments
}

\section{Empowerment by Capacity Building in Urban Quarters - First Results and Assessment of a New Standardised Instrument}

Autoren

Institut

\section{A. Trojan, S. Nickel}

Universitätsklinikum Hamburg-Eppendorf, Zentrum für Psychosoziale Medizin, Institut für Medizin-Soziologie
Schlüsselwörter

- Kapazitätsentwicklung

- soziale Benachteiligung

- Gesundheitsförderung

- Quartiersentwicklung

- Empowerment

Key words

- capacity building

- social disadvantage

- health promotion

- community development

- empowerment

\section{Bibliografie}

DOI 10.1055/s-0028-1103306 Gesundheitswesen 2008;

70: 771-778

(c) Georg Thieme Verlag KG Stuttgart · New York

ISSN 0941-3790

\section{Korrespondenzadresse}

Dr. phil. S. Nickel

Universitätsklinikum HamburgEppendorf

Zentrum für Psychosoziale Medizin

Institut für Medizin-Soziologie Martinistraße 52

20246 Hamburg

nickel@uke.uni-hamburg.de

\section{Zusammenfassung}

$\nabla$

Ziele: Kapazitätsentwicklung („Capacity Building“) gilt als intermediäre Ziel- und Erfolgsgröße für gemeindenahe Gesundheitsförderung und Empowerment. Erstes Ziel der Studie war die Entwicklung und Erprobung eines Erhebungsinstruments zur Messung dieses Konzepts im Rahmen eines Programms zur Gesundheitsförderung bei Kindern und Eltern in einem sozial benachteiligten Quartier (ca. 3000 Bewohner, $60 \%$ mit Migrationshintergrund). Zweites Ziel war die retrospektive Ermittlung der Kapazitätsentwicklung in einem Fünfjahreszeitraum.

Methodik: Es wurden standardisierte Befragungen bei 27 Akteuren aus dem Gesundheitsund Sozialbereich durchgeführt. Themen der Befragung waren: Bürgerbeteiligung, lokale Führung, vorhandene Ressourcen, Vernetzung und Kooperation, Unterstützung der Bewohner. Gleichzeitig wurde erprobt, inwieweit das Instrument für eine Bewohnerbefragung geeignet ist. Außerdem wurden Vorformen des Instruments und die Ergebnisse im Hinblick auf Verbesserungsmöglichkeiten mit einem Expertenbeirat diskutiert.

Ergebnisse: Der Erfolg des Vorhabens besteht neben der praktischen Anwendung der Ergebnisse im Quartier darin, dass ein psychometrisch geprüftes Instrument zur Messung von Kapazitätsentwicklung entstand und wichtige Erfahrungen mit der Durchführbarkeit solcher Studien gemacht wurden. Im Vergleich der Situation im Jahr 2006 mit der 2001 zeigten sich deutliche Verbesserungen im Quartier auf allen Dimensionen der Kapazitätsentwicklung.

Schlussfolgerung: Die langfristige Etablierung des Instruments erfordert in Zukunft eine intensivere Auseinandersetzung nicht nur mit den Chancen, sondern auch mit den Grenzen des Instruments. Die positive Fünfjahresbilanz ist vor allem auf das allgemeine Klima der Quartiersent-

\section{Abstract}

V

Aims: Capacity building is regarded as an intermediate outcome and success parameter for community health promotion and empowerment. The first aim of the study was the development and examination of a tool to measure capacity building in the context of a programme of health promotion for children and parents in a socially disadvantaged quarter in Hamburg (about 3000 residents, $60 \%$ with migration background). The second aim was to measure capacity building within a period of five years.

Method: A survey was carried out with 27 professionals from the health and social field. Dimensions of the instrument were: citizen participation, local leadership, available resources, networking and co-operation, support of residents. At the same time, we tested to what extent the instrument is suitable for resident surveys. Furthermore, pretest instrument and results were discussed with our advisory board regarding potential improvements.

Results: The result of the project, in addition to its practical use in the quarter, consists of the generation of a psychometrically tested instrument to measure capacity building. Furthermore, the experience contributed to the expertise on how such a study can be conducted. A comparison of the situation in 2006 with the situation in 2001 showed considerable improvements in all dimensions of capacity building in the quarter.

Conclusion: Establishing the instrument on a long-term basis calls for more intensive examination not only of the chances, but also the limitations of the tool. We mainly attribute the overall positive picture that was gained within a period of five years to the generally supportive climate of development in the quarter as well as to the strong and continuous commitment of individual persons involved. 
wicklung und Gesundheitsförderung sowie auf das starke Engagement und die Beharrlichkeit einzelner Akteure zurückzuführen.

\section{Einleitung}

Strukturbildung (Capacity Building) als Globalkonzept für intermediäre Erfolgsmessung

Strukturbildung (bzw. Capacity Building) ist in Deutschland bisher kaum als Konzept genutzt worden. Viel bekannter ist das inhaltlich ähnliche Konzept Empowerment (siehe weiter unten). Seit einigen Jahren wird Strukturbildung von Walter und Schwartz [1] explizit als Nutzendimension und Zielparameter für Prävention und Gesundheitsförderung aufgeführt. Die Erläuterung zu diesem Zielparameter lautet (S. 206):

„Als Schätzer für die Dauerhaftigkeit angestoßener Entwicklungen gilt das sog. Capacity Building. Dieser relativ neue - in der Literatur noch unterschiedlich weit gefasste - Indikator bezieht sich im Wesentlichen auf die Bereitschaft und Befähigung, geeignete Strukturen zu entwickeln, um eine spezifische Maßnahme erfolgreich anzubieten und aufrecht zu erhalten, bis hin zur Nutzung und Mobilisierung geeigneter Ressourcen und Kooperationsstrukturen und der Entwicklung adäquater Strategien zur Umsetzung und Implementierung von Maßnahmen“.

Aus dieser Erläuterung wird die Komplexität des Konzeptes deutlich, aber auch seine Eignung als intermediärer Erfolgsindikator für setting-bezogene Gesundheitsförderungsansätze.

Als Quelle wird von Walter und Schwartz auf Hawe u.a [2]. verwiesen, die im Rahmen einer Studie mit sechs Fokusgruppen von Gesundheitsförderern die Bedeutung dieses Begriffes und die Erfahrungen mit Capacity Building exploriert haben. Ausgangspunkt für die Studie von Hawe u.a. war ein Überblick, in dem drei wesentliche Aspekte dieses Konzepts identifiziert wurden, und zwar:

- Aufbau von Gesundheitsstrukturen und Gesundheitsleistungen (einschließlich Planung, Evaluationsfähigkeiten und Ressourcen),

- Programmaufrechterhaltung und Nachhaltigkeit sowie

- Problemlösungsfähigkeiten von Organisationen und Gemeinschaften.

In der Gesundheitsförderungsstrategie der WHO taucht der Ausdruck (Community) Capacity Building das erste Mal in der Jakarta-Erklärung „Gesundheitsförderung für das 21. Jahrhundert“ auf. Als vierte Priorität wird dort genannt: „Increase community capacity and empower the individual“ (deutsch „Kompetenzen der Gemeinden und die Befähigung der Einzelnen fördern“). Der Akzent liegt im weiteren erläuternden Text vor allem auf dem Aufbau von Partnerschaften für Kooperation und der Mobilisierung von Wissen, Fähigkeiten und Ressourcen. In dem kurze Zeit darauf erschienenen „Health Promotion Glossary“ [3] hat Capacity Building keinen eigenständigen Eintrag (ebenso wenig wie in einem Grundsatzreferat über die Evaluation von Gesundheitsförderung [4]), wird aber im Kontext der intermediären Gesundheitsergebnisse erwähnt: In diesem Zusammenhang wird von „partizipativen“ und „pluralistischen“ Evaluationsansätzen gesprochen, „which may help building capacities of individuals, communities, organizations and governments to address important health problems“.

In einer Ergänzung des WHO-Glossars [5] wird Capacity Building explizit aufgenommen, jedoch nicht von dem eng verwand- ten Konzept Empowerment abgegrenzt. Die Formulierungen legen vielmehr nahe, dass Aktivitäten und Prozesse des Capacity Building wie auch des Empowerment [6,7] letztendlich zu denselben Ergebnissen führen, nämlich zu Gemeinden bzw. Quartieren, die fähig sind, „to take action to address their needs as well as the social and political support that is required for successful implementation of programs“ (so die Formulierung für Capacity Building bei Smith et al. [5]). Man könnte als Unterschied allenfalls unterstellen, dass Empowerment mehr die politische Befähigung Benachteiligter anspricht, während der Akzent bei „knowledge, skills, commitment, structures, systems and leadership“ als Kernelemente von Capacity Building mehr auf den pragmatischen Lernprozess eines Gemeinwesens fokussiert.

Goodman et al. [8] haben als Ergebnis einer Konsensus-Konferenz zehn für das Konzept des Capacity Building zentrale Dimensionen ermittelt. Diese sollten nach ihrer Empfehlung aber lediglich als Ausgangspunkt dienen, das Konzept zu erweitern, zu präzisieren und zu messen. Überlappungen mit anderen Konzepten ${ }^{1}$ und der Mangel an angemessenen Messmethoden werden ausdrücklich festgestellt.

Wir sind in unserem Projekt von einer schon empirisch erprobten Einteilung von Laverack und Labonte [12] mit 9 Dimensionen ausgegangen (vgl. dazu auch Laverack G, „Messung, Bewertung und strategische Weiterentwicklung von Gemeindekapazität und -empowerment“ in diesem Heft). In einem folgenden Beitrag [13] wurde das Konzept weiter ausgearbeitet und festgestellt, dass die Messung andere Erfolgsparameter ergänzt (population health indicators; programme-specific indicators), insbesondere dann, wenn Veränderungen der Endpunkt-Evaluation nur langfristig zu erwarten sind.

Ein unmittelbar anknüpfender Beitrag von Labonte \& Laverack [14] stellt die Frage nach geeigneten Messmethoden. Dabei werden verschiedene Ansätze ins Auge gefasst, insbesondere Experteninterviews, Fokusgruppen, Surveys, Dokumentations- und Sekundäranalysen. Wer die Einschätzungen der Ausgangssituation und der späteren Veränderungen vornimmt, wird nicht genau festgelegt. In jedem Fall soll es die Gemeinde aber möglichst weitgehend selbst tun. Für die Visualisierung werden Beispiele aus ersten Feldtestungen in Form von Spinnennetzdiagrammen angeführt [15].

Die Analyse der internationalen Literatur zu Beginn unseres Projektes zeigte, dass „Capacity Building“ zunehmend größere Aufmerksamkeit erfuhr und ein Konsens über die für die Evaluation relevanten Dimensionen weitgehend erzielt war, dass aber für die Messung keine etablierten Verfahren bestanden. In Deutschland war das Konzept als intermediärer Erfolgsparameter zwar rezipiert und akzeptiert worden, wurde allerdings noch nicht angewendet und wissenschaftlich bearbeitet.

Diese Lücke versucht unser Projekt zu füllen. Nähere Informationen dazu sind an anderen Stellen publiziert worden $[16,17,18]$.

Im Folgenden werden zunächst die Methoden dargestellt und Ergebnisse der Studie berichtet. Daran anschließend werden in der Diskussion vor allem zwei Punkte angesprochen:

${ }^{1}$ Im Kontext dieses Beitrages kann nicht ausführlicher eingegangen werden auf die vielfältigen Überlappungen und Verwandtschaften von Capacity Building mit benachbarten Konzepten, insbesondere den mit Community verknüpften Begriffen Development [9], Organisation and Building [10], Participation [11] und den ebenfalls überlappenden Begriffen von Coalition Building, Social Capital, Social Networking, Social Mobilisation [4] - um nur die wichtigsten zu nennen. 
- Gemeinsamkeiten und Unterschiede unserer Messung des Konzepts im Vergleich mit der Literatur und

- mögliche Funktionen des Konzepts und eine Einschätzung, inwieweit sie mit unserem Instrument erfüllt werden können.

\section{Methode \\ $\nabla$}

Die Untersuchung fand statt in einer dicht bewohnten Hochhaussiedlung in Hamburg-Lokstedt, einem von neun Stadtteilen des Bezirks Eimsbüttel, mit der Kurzbezeichnung „Lenzsiedlung“. Seit 2000 war das Wohnquartier ein Gebiet des Hamburger Senatsprogramms zur Sozialen Stadtteilentwicklung (seit 2005: Aktive Stadtteilentwicklung) und blieb es bis Ende Februar 2007. Nach den aktuellen Daten leben hier insgesamt 3085 Menschen, davon sind rund 53\% weiblich und rund 29\% unter 18 Jahren. Zudem leben in der Lenzsiedlung zu fast $60 \%$ Menschen mit Migrationshintergrund. Eine weitere Besonderheit des Quartiers besteht darin, dass jeder dritte Einwohner der Lenzsiedlung „Hartz-IV-Leistungen“ erhält. 2005 startete ein vom Gesundheitsamt moderiertes Programm zur Gesundheitsförderung bei Kindern und Eltern, in dem verschiedene Einrichtungen in einem „Runden Tisch“ zusammenarbeiten und das auf 7-10 Jahre angelegt ist.

Informanten über die „Community Capacities“ waren in der Studie sowohl professionelle Akteure aus dem Gesundheits- und Sozialbereich als auch sozial engagierte und über den Stadtteil gut informierte Bewohner aus der Lenzsiedlung. Die Akteurbefragung der Professionellen war als schriftliche „Vollerhebung“, d.h. als Befragung möglichst aller relevanten Akteure aus dem professionellen Bereich angelegt (Professionellen-Befragung). Demgegenüber sollte die ebenfalls schriftlich angelegte Bewohnerbefragung eine Auswahl von als „aktiv bekannten“ Personen umfassen: Mitglieder des Stadtteilbeirats, Aktive in lokalen Projekten sowie Personen, die sich im Rahmen einer vorherigen Passantenbefragung besonders interessiert gezeigt haben [17].

\section{Erhebungsinstrument}

Bei dem verwendeten Erhebungsinstrument handelte es sich um eine Neuentwicklung auf Basis der beschriebenen Vorarbeiten von Laverack und Labonte $[12,13]$ sowie weiterer internationaler Studien und Übersichtsarbeiten [2,6,19-22]. Nach einer vertiefenden Diskussion der Literatur im Forscherteam und einer explorativen Beurteilung durch neun Experten aus Wissenschaft und Praxis im Umfeld des untersuchten Präventionsprogramms in der Lenzsiedlung wurde eine erste Version des Instruments erstellt. Die Anpassungen an deutsche Verhältnisse sind vor allem als Konkretisierung, Bündelung und Akzentuierung der ursprünglichen Themenbereiche wie auch deren Anwendbarkeit auf die Quartiersebene zu sehen ${ }^{2}$. Der so entstandene Fragebogen zur „Kapazitätsentwicklung im Quartier“(KEQ) umfasst fünf Themenkomplexe bzw. Dimensionen, die mit 54 geschlossenen Fragen (5-Punkte-Skalen) operationalisiert wurden:

\footnotetext{
${ }^{2}$ Auch andere Autoren erwähnen Überlappungen der neun Bereiche und daraus resultierende „Verdichtungen“ der Dimensionen (s. NSW Health 2001, Laverack 2008 b).
}

- Bürgerbeteiligung (Ausmaß der Bürgerbeteiligung, Eigeninitiative der Bewohner, Förderung der Bürgerbeteiligung),

- lokale Führung (Ausmaß lokaler Führung, soziale Kompetenzen und Steuerungskompetenzen der lokalen Führung),

- vorhandene Ressourcen (materielle Ressourcen, Wissen und Informationen, soziale Ressourcen),

- Vernetzung und Kooperation (örtliche und überörtliche Vernetzung und Kooperation, Qualität der Vernetzung und Kooperation) und

- Unterstützung der Bewohner (Unterstützung durch Ämter/ Behörden, „Brückeneinrichtungen“ und professionelle Dienste).

In der aktuellen, weiterentwickelten Fassung des Instruments wurde die letzte Dimension um verschiedene Angebote zur Förderung und zum Schutze der Gesundheit von Menschen erweitert und in „Gesundheitsversorgung“ umbenannt. Im Folgenden werden wir jedoch die in der Pilotstudie verwendete Bezeichnung der Dimension beibehalten.

\section{Durchführung der Befragung \\ $\nabla$}

Die Durchführung der Erhebung erfolgte zwischen Juni und Juli 2006. Neben der Einschätzung der aktuellen Situation (T1) sollte auch eine retrospektive Einschätzung der Situation vor der Einführung einzelner Gesundheitsförderungsangebote im Mai 2001 (T0) erfolgen. Zusätzlich zum Grad der Zustimmung wurden auch qualitative Angaben erbeten, aus denen hervorgeht, warum die Statements als mehr oder weniger zutreffend eingeschätzt wurden. Ausgehend von einer Liste, die gemeinsam mit unseren Praxispartner erstellt wurde, haben wir für die Professionellen-Befragung 48 Akteure aus dem Gesundheits- und Sozialbereich (u.a. Stadtteilentwicklung, Gemeinwesenarbeit, Bildung und Erziehung) im Umfeld der Lenzsiedlung zur Mitwirkung an der Befragung aufgerufen. Nach Zusendung bzw. Aushändigung der Befragungsunterlagen wurden aufgrund von nachträglichen Absagen und/oder kurzfristiger Nichterreichbarkeit tatsächlich nur 27 Fragebögen bei der Auswertung berücksichtigt (56\% Ausschöpfungsquote).

Der Versuch, auch sozial engagierte Bewohner in etwa gleich großer Anzahl im Rahmen der Bewohnerbefragung zu dieser Thematik zu befragen, hat sich indes als nicht praktikabel herausgestellt: Trotz vielfacher Kontaktversuche und Unterstützungsangebote haben letztlich nur 6 von 20 als aktiv bekannte Bewohner an der Befragung teilgenommen; zudem fühlten sich die Antworter oft nicht kompetent genug, alle Fragen zu beantworten. Im systematischen Vergleich der vorliegenden Antworten ließen sich allerdings keine nennenswerten Unterschiede zu den Professionellen auffinden.

\section{Ergebnisse}

$\nabla$

In diesem Abschnitt werden die Ergebnisse zur Entwicklung von nachhaltigen Strukturen und Kapazitäten aus Sicht der professionellen Akteure dargestellt. Die quantitativen Ergebnisse, ergänzt durch die qualitativen Angaben im Fragebogen, geben Aufschluss darüber, was „Schwachstellen“ oder „Stärken“ der Entwicklung im 5-Jahres-Zeitraum gewesen sind. Zur besseren Übersicht werden die Ergebnisse nach ihrer Erhebungstiefe dar- 


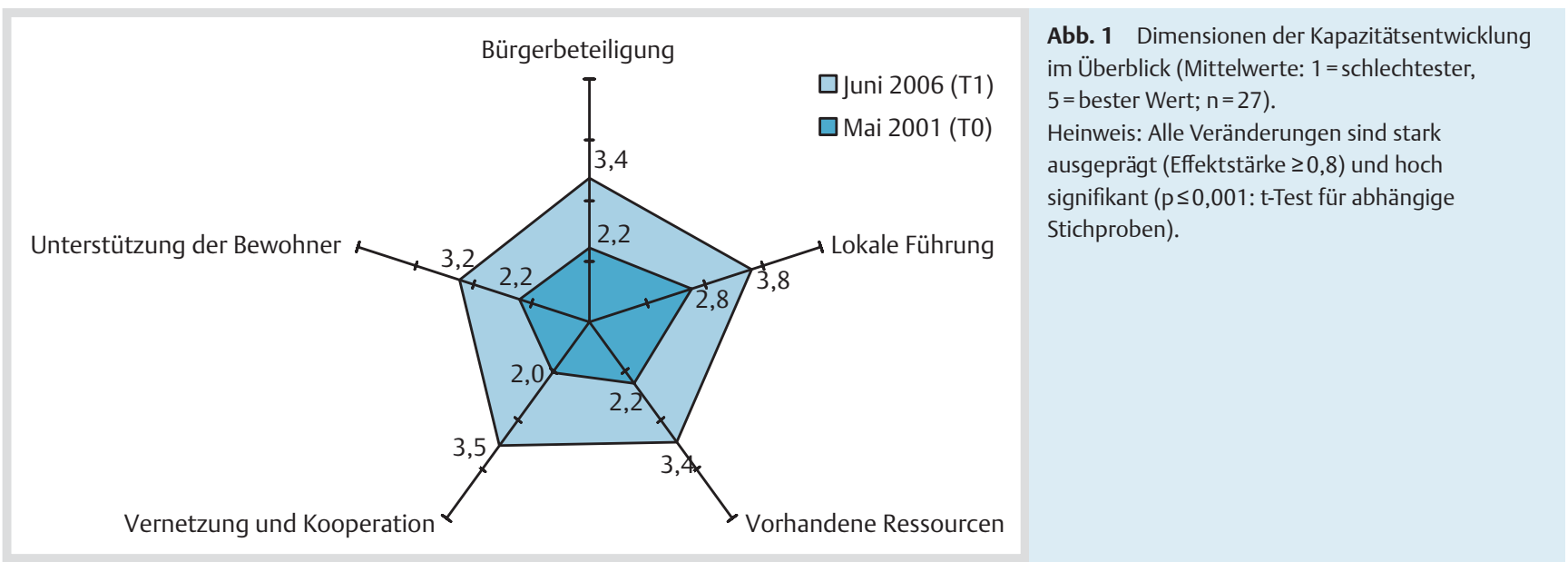

gestellt: angefangen von den Dimensionen bis hin zu den Einzelkriterien der Kapazitätsentwicklung.

\section{Überblick zu den Dimensionen \\ $\nabla$}

Die Ergebnisse für die fünf Dimensionen von Kapazitätsentwicklung sind in einem Netzdiagramm zusammengefasst (vgl. - Abb. 1). Dargestellt werden die durchschnittlichen Punktwerte pro Dimension in 2001 und 2006, die jeweils von 1 bis 5 ( = Bestwert) reichen können. Ein positives Vorzeichen der Effektstärke (siehe Erläuterungen in den Tabellenlegenden) weist immer auf eine Verbesserung der Situation zwischen 2001 und 2006 hin, ein negatives auf eine mögliche Verschlechterung. Für die Stadtteilentwicklung und Gesundheitsförderung im Quartier sind zunächst die insgesamt äußerst positiven Ergebnisse zu betonen: Diese können vor allem auf das allgemeine „Klima“ der Quartiersentwicklung und Gesundheitsförderung sowie auf das starke Engagement und die Beharrlichkeit einzelner Akteure aus dem gesundheitlichen und sozialen Sektor zurückgeführt werden. Die positiven Beurteilungen in den Bereichen „lokale Führung“ $(3,8)$, „Vernetzung und Kooperation“ $(3,5)$ sowie in Einzelaspekten der Subdimension „Unterstützung durch Brückeneinrichtungen“ (zwischen 3,5 und 4) hängen sicher vorwiegend mit der geleisteten Informations-, Koordinations- und Aktivierungsarbeit der beiden Infrastruktursäulen Lenzgesund e.V. und Stadtteilbüro der „Sozialen Stadtteilentwicklung“ (seit 2005 „Aktive Stadtteilentwicklung“) zusammen. Ein Vergleich der Professionellen aus dem Sozial- und Gesundheitsbereich ergab, dass sich die Ergebnisse nur in Einzelfällen unterscheiden. Die Expertenmeinungen scheinen demnach relativ homogen zu sein.

\section{Einzelkriterien der Kapazitätsentwicklung}

Betrachtet man die Rangliste der 10 am besten bewerteten von den 54 Einzelkriterien im Jahr 2006, so fällt auf, dass viele Ziele des Hamburger Programms zur Sozialen Stadtteilentwicklung (u.a. Verbesserung des Wohnumfelds durch Erneuerung/Erweiterung der Grün- und Spielflächen, Förderung bürgerschaftlichen Engagements, bessere Informationen über stadtteilbezogene Aktivitäten und Angebote) bereits in fünf Jahren erreicht werden konnten (vgl. @ Abb. 2). Positive Veränderungen finden sich ansonsten vor allem in den Teilaspekten der „lokalen Führung“. Der stärkste Fortschritt auf dieser Dimension besteht darin, dass Ämter und soziale Einrichtungen wie etwa die Kindertagesstätten (Kitas) zunehmend ihre Verantwortung für die Lenzsiedlung erkennen und annehmen. Hinsichtlich der Qualitäten der Führungspersonen haben sich ebenfalls deutliche Verbesserungen ergeben: Diese betreffen sowohl die wahrgenommenen sozialen Kompetenzen (z.B. demokratischer und integrativer Führungsstil) als auch die Steuerungskompetenzen (z.B. Dokumentation von Aktivitäten in der Lenzsiedlung).

Auf einzelne Schwachstellen und den Handlungsbedarf für Kapazitätsentwicklung muss intensiver eingegangen werden, weil ein Ziel der Befragung ja gerade in der Identifikation von Ansatzpunkten liegt, die man gezielt verbessern möchte (vgl. - Abb. 3). Relativ kritische Beurteilungen erhielten demnach vor allem Kriterien, die auf die Motivierung und Qualifizierung von Bewohnern aus dem Quartier abzielen, um auch selbstständig Initiativen zu ergreifen und/oder mehr Eigenverantwortung zu übernehmen (vorrangig im niedrigschwelligen Bereich, wie z.B. bei der Kinderbetreuung oder Festvorbereitung); hier gilt es nicht zuletzt, die gewöhnlich kleine Gruppe von Bewohnern, die bereit und in der Lage ist, dauerhaft in Bürgerinitiativen und Projekten mitzuwirken, durch geeignete Dialogformen stärker zu unterstützen. Im Vergleich zu anderen professionellen Diensten steht auch die Unterstützung durch die ansässigen Ärzte eher noch am Anfang: Einige engagieren sich zwar verstärkt für die Bewohner im Quartier (z.B. durch Informationsveranstaltungen, Teilnahme am „Runden Tisch“), doch gibt es kein gemeinschaftliches Vorgehen und wenig Öffnung der bestehenden Angebote (z.B. Unterstützung der Gruppenarbeit von gefährdeten oder kranken Menschen). Diese und andere Ergebnisse (u.a. negative Außenwahrnehmung des Quartiers, unzureichende Finanzierungsmöglichkeiten von Projekten) sind in die Formulierung von Handlungsempfehlungen für das weitere Quartiersmanagement in der Lenzsiedlung eingegangen.

\section{Diskussion und Schlussfolgerungen \\ $\nabla$}

Das Erhebungsinstrument und die Ergebnisse in unserem Untersuchungsfeld, der Hamburger Lenzsiedlung, werfen vielfältige Fragen auf. An dieser Stelle geht es vorrangig um eine vorläufige Einschätzung des Erhebungsinstruments. Die hierzu folgenden Überlegungen gehen einerseits auf die Auseinandersetzung mit 


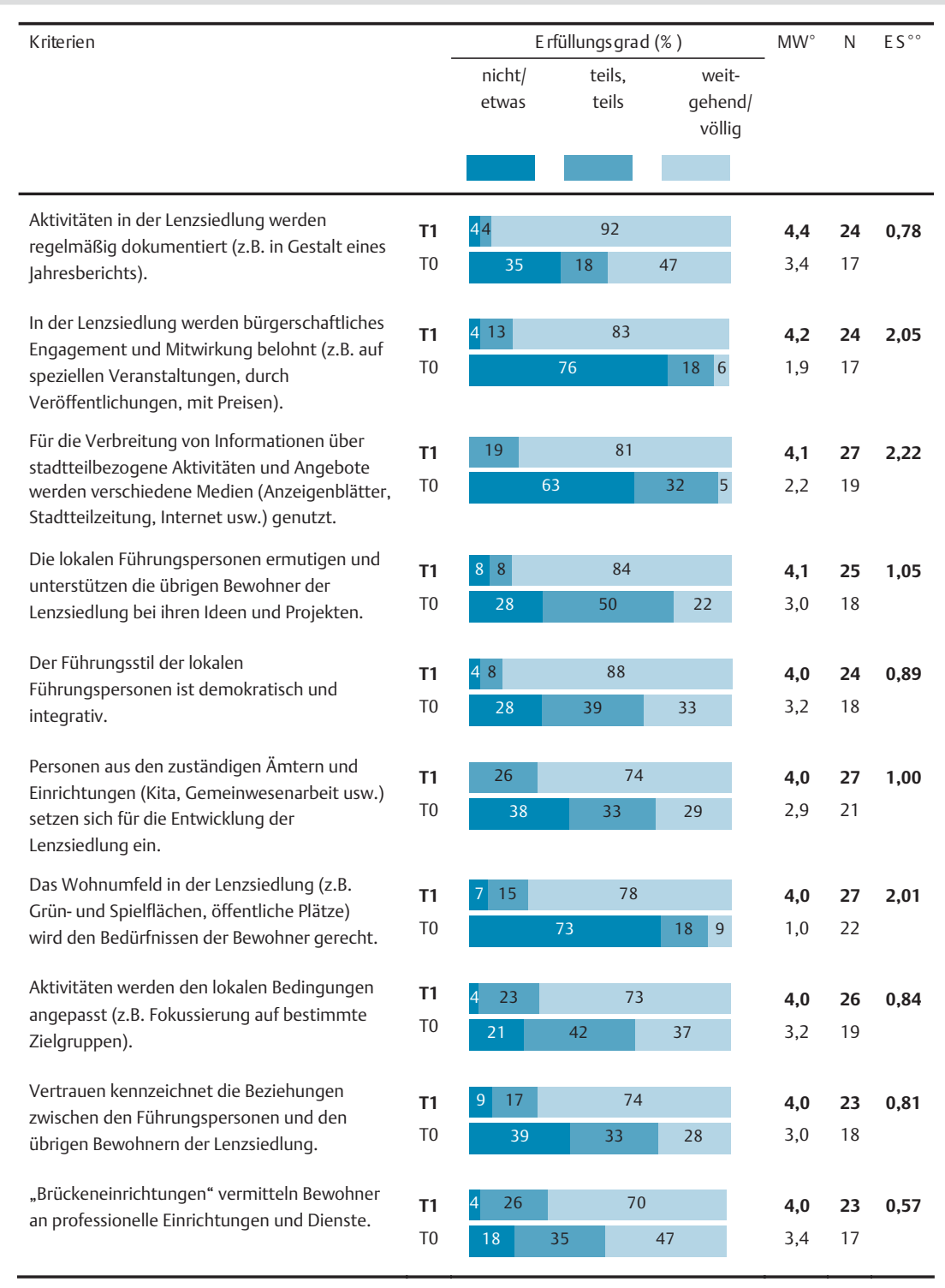

Abb. 2 TOP-10 der besten Einzelkriterien für Kapazitätsentwicklung in 2006 (MW = Mittelwert; $\mathrm{N}=$ Anzahl der Antworter; $\mathrm{ES}=$ Effektstärke). ${ }^{\circ}$ Skalenbereich: 1 bis 5 (Bestwert); ${ }^{\circ} \mathrm{ES} \geq 0,2$ "kleiner“, $\geq 0,5$ "mittlerer“, $\geq 0,8$ "großer“ Effekt. den bekannten Vertretern der Verwendung des Erfolgsparameters „Capacity Building“ zurück wie auch auf Diskussionen mit unserem Forschungsbeirat, in dem auch die wichtigsten Praxisvertreter des Untersuchungsfeldes repräsentiert sind ${ }^{3}$.

\section{Wie stellen sich unsere Verwendung des Konzepts und} seine Messung im Vergleich zu der von Laverack, einem der maßgeblichen Gestalter, dar?

Inhaltlich gibt es keine großen Unterschiede, obwohl wir nur fünf statt der von Laverack vorgeschlagenen neun Dimensionen für den Aufbau des Instruments genutzt haben. Auch das Verhältnis zu (Community) Empowerment ist gleichermaßen eng. Beide Konzepte benennen eigentlich Handlungsprozesse und keine Zustandsbeschreibungen. Um den Erfolg dieser Handlungsprozesse zu beschreiben, untersucht man, inwieweit in einem Gemeinwesen Strukturen und Kompetenzen einen $\mathrm{Zu}$ stand erreicht haben, der den Bewohnern erlaubt, Kontrolle

${ }^{3}$ Dipl.-Ing. Christa Böhme, Institut für Urbanistik (difu); Prof. Eberhard Göpel, Hochschule Magdeburg-Stendal; Dipl.-Psych. Dipl. Pol. Thomas Kliche, UKE, Institut und Poliklinik für Medizinische Psychologie; Dr. Michael T. Wright, Wissenschaftszentrum Berlin für Sozialforschung (WZB). über ihre eigene Gesundheit und die Determinanten der Gesundheit zu gewinnen. Weitere Elemente, die wir von der Arbeitsgruppe um Laverack übernommen haben, sind die qualitativen Begründungen für die Einschätzungen auf einer Stufenleiter sowie die spätere Visualisierung durch Spinnennetzdiagramme. Gemeinsam ist auch, dass es nicht primär um die zu einem bestimmten Zeitpunkt gemessenen absoluten Werte geht, sondern vielmehr um eine kontinuierliche Fortschrittsmessung, die den Prozess der Gesundheitsförderung in einem Gemeinwesen anleiten und steuern soll.

Es gibt jedoch auch Unterschiede: Als Kernziel erkennen wir bei Laverack [23], Beteiligungsmöglichkeiten für eine Bewohnerschaft im Rahmen von Top-Down-Programmen zu schaffen, d.h. Hauptelement in der Strategie des „Parallel Tracking“ zu sein (vgl. dazu auch Laverack G, „Berücksichtigung des Empowerment in der Programmplanung von Gesundheitsförderung“ in diesem Heft). Kernziel in unserem Projekt hingegen war ursprünglich, einen globalen Erfolgsparameter settingbezogener Gesundheitsförderung quantitativ zu messen.

Der Anspruch, ein Instrument quantitativer Messung zu entwickeln, stellt uns jedoch auch vor besondere methodische Probleme: Während bei Laverack eine undefinierte Anzahl und $\mathrm{Zu}$ - 


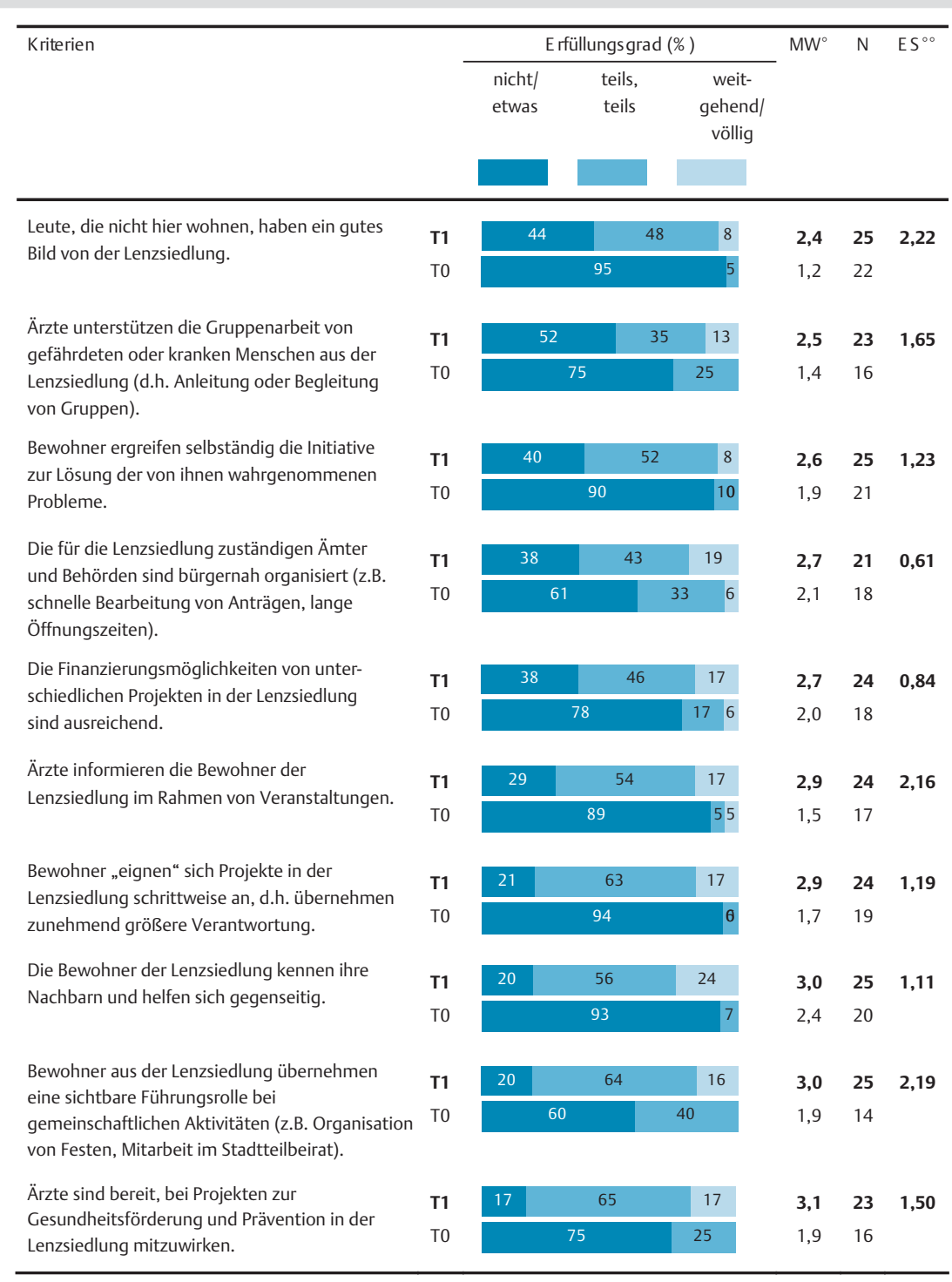

Abb. 3 TOP-10 der schlechtesten Einzelkriterien für Kapazitätsentwicklung in 2006 (MW = Mittelwert; $N=$ Anzahl der Antworter; $\mathrm{ES}=$ Effektstärke).

${ }^{\circ}$ Skalenbereich: 1 bis 5 (Bestwert); ${ }^{\circ} \mathrm{ES} \geq 0,2$ „kleiner“, $\geq 0,5$ "mittlerer“, $\geq 0,8$ "großer“ Effekt. sammensetzung von „Bewohnern“ bzw. „Repräsentanten der Community“ ausreicht, um Einschätzungen (methodisch am besten als Fokusgruppe zu charakterisieren) vorzunehmen, benötigen wir eine genügend große Anzahl von Kennern der Community mit möglichst breiten, über ihre jeweiligen Spezialinteressen hinausgehenden allgemeinen Informationen über den Stadtteil. Diese sind nicht unter allen Gegebenheiten zu finden. In der vorliegenden Pilotstudie ist uns dies für professionell beteiligte Akteure in der Lenzsiedlung gelungen, für die Bewohnerschaft hingegen nicht in genügendem Umfang.

\section{Erhebungsinstrument für Evaluation oder für kontinuierliche Qualitätsentwicklung?}

Als Evaluationsinstrument hat der KEQ-Fragebogen unseres Erachtens zwei zentrale Probleme. Dadurch, dass eine quantitativ auswertbare Bewohnerbefragung „mangels Masse“ nicht zustande kam, ist das Ergebnis eine expertenbasierte Evaluation, wobei die Experten ganz stark in den Prozess involviert sind und daher letztlich ihre eigene Arbeit bewerten. (Dies kann allerdings sowohl zu positive als auch zu negative Einschätzungen ergeben, letzteres aufgrund überhöhter Erwartungen an die Schnelligkeit von Veränderungsprozessen.) Auch in unserem Ar- beitszusammenhang haben wir das Instrument von vornherein als Baustein in einem multimethodischen Gesamtkonzept der Evaluation aufgefasst. In diesem Gesamtkonzept geht es um eine Triangulierung sowohl der Zugänge und Methoden [17]. Dies entspricht dem allgemeinen Konsens über die Notwendigkeit, verschiedene Instrumente und Methoden für die Evaluation von Gesundheitsförderung einzusetzen [4,24-27].

Das zweite Problem besteht in dem Anspruch, ein generisches Instrument für Kapazitätsentwicklung darzustellen. Der hierdurch nötige Abstraktionsgrad der Statements ist problematisch. Hinzu kommt, dass die Akzentuierung des Instruments weiterer Diskussion bedarf: Inwieweit dürfen allgemeine Themen der Gemeinwesenentwicklung und -partizipation die konkret auf Gesundheit bezogenen Themen dominieren? In welchem Umfang können und sollen Determinanten der Gesundheit aus anderen Politiksektoren, z.B. Stadtentwicklung, Soziales, Jugend, explizit erhoben werden? Auf welche Größenordnungen von Gemeinwesen passt das Instrument, das derzeit für Quartiere und Stadtteile zwischen ca. 3000 und 30000 Einwohnern verwendet wird? - Alle diese Fragen entstehen im Prinzip nur deswegen, weil das Instrument nicht nur einmalig die Zielerreichung eines spezifischen Programms prüfen soll, sondern mög- 
lichst in allen Zusammenhängen nutzbar sein soll, bei denen es um multimodale Gesundheitsförderung im Setting Gemeinde geht. Dies zu überprüfen, ist eine Forschungsfrage, bei der wir erst am Anfang stehen ${ }^{4}$.

Einfacher zu erfüllen ist der Anspruch, ein Instrument der Qualitätsentwicklung zu sein: Die expertenbasierte Beurteilung der fünf Dimensionen bzw. „Domänen“ von Strukturbildung und Kompetenzentwicklung in einem Stadtteil entspricht weitgehend einer Selbstbewertung bzw. einem Selbstaudit zu Beginn eines Qualitätsentwicklungsprozesses. Dabei spielt sowohl die Einstufung der Qualität auf bestimmten Dimensionen und in bestimmten Parametern eine Rolle als auch die Erläuterung und Darlegung in den qualitativen Fragen des Erhebungsinstrumentes. Mithilfe des Forschungsprojektes ist eine Quartiersdiagnose entstanden, die im Prinzip auch als Qualitätsbericht (mit internen und externen Bewertungselementen) bezeichnet werden könnte [16]. Zusammen mit einem spezifischer auf die Kindergesundheit in der Lenzsiedlung ausgerichteten zweiten Bericht [18] ist für die Lenzsiedlung eine Standort- und Potenzialbestimmung erfolgt, die auch unmittelbar zur Überarbeitung und Anpassung des Präventionsprogramms in dem Viertel geführt hat ([18] S. $61 \mathrm{ff}$.).

Mit der Einordnung als Selbstbewertung im Rahmen eines Optimierungsparadigmas (statt eines Evaluationsparadigmas im Sinne der Evidenzschaffung) kommt die Einordnung auch wieder dem ursprünglichen Ansatz von Laverack u.a. als Partizipations-,,Tool“ im „parallel tracking“ näher. Diese Sichtweise würde auch nahelegen, das generische Verfahren der Qualitätsentwicklung mit KEQ extern mit den am besten etablierten anderen Qualitätsentwicklungsinstrumenten im Sinne einer Fremdbewertung (in erster Linie mit QIP-Qualität in der Prävention [27]) oder dem Qualitätsentwicklungsverfahren der Gesundheitsinitiative „Gesund. Leben. Bayern.“ [26] zu validieren.

\section{Kann KEQ den Funktionen von Evaluationen gerecht werden?}

Wir denken, dass trotz der geschilderten Fokussierung des Instruments auf kontinuierliche Qualitätsentwicklung es im weiten Sinne auch evaluative Aufgaben erfüllt. Stockmann [28] beschreibt vier wesentliche Funktionen von Evaluationen, auf die wir im Folgenden kurz eingehen wollen.

- Erkenntnisfunktion:

Zweifellos hat das Instrument für das untersuchte Feld relevante Erkenntnisfunktionen gehabt und teilweise sogar „AhaErlebnisse" ausgelöst. Die Verbreiterung des Wissens von Problemen und Problemlösungsmöglichkeiten des Viertels kann man per se als Beitrag zum Capacity Building betrachten. Eine weiterreichende Frage aber richtet sich darauf, ob das Instrument geeignet ist, unsere Wissensbasis (Evidenzbasis) über die Wirkungen von multimodalen Programmen der Gesundheitsförderung im Setting Gemeinde zu verbreitern. Hierüber lässt sich erst etwas sagen, wenn das Programm in verschiedenen programmatischen und geografischen Zusammenhängen weiter erprobt ist.

- Kontrollfunktion:

Eng mit der vorangehenden Funktion verknüpft ist die (Selbst-) Kontrolle der Quartiersentwicklung im Sinne einer Stärkung von Kompetenzen und Strukturen für mehr Gesundheit. Da-

\footnotetext{
${ }^{4}$ Wir sind interessiert, das Instrument in anderen Gebieten mit multimodalen Gesundheitsförderungsprogrammen zu erproben. Kontaktaufnahme über die Autoren!
}

bei wäre wünschenswert, dass die jetzige Selbstkontrolle der Experten ergänzt wird durch eine vereinfachte, aber auf gleiche Dimensionen abzielende Kontrolle des Programms durch die Bewohner. Unmittelbare Befragungen von Bewohnern sind zwar auch schon Teil des Projektes gewesen, sie waren aber nicht in direkter Weise auf eine Überprüfung der Experteneinschätzungen ausgerichtet.

- Dialogfunktion:

Auch diese lässt sich für die Professionellen als erfüllt einschätzen. Für die Bewohner gilt dies hingegen nur punktuell. Eine interessante und wichtige Erweiterung wäre aber, wenn es andere mit demselben generischen Instrument untersuchte Stadtteile geben würde, mit denen sich die Lenzsiedlung im Sinne eines Benchmarkings vergleichen kann. (Hierzu laufen Untersuchungen und Versuche, das Instrument in Programmen einzusetzen, die in unterschiedlichen Gebieten durchgeführt werden.) Der Runde Tisch „Lenzgesund“ hat mehrfach die Ergebnisse diskutiert. Allerdings ist der Dialog mit der Bewohnerschaft bisher nur punktuell, nicht jedoch auf breiter Fläche gelungen.

- Legitimationsfunktion:

Diese Funktion wird auch von Laverack [23] ausdrücklich als wichtiges Teilziel der Erhebung von Capacities in einer Gemeinde angegeben. Tatsächlich stellen Berichte wie die Quartiersdiagnose oder integrierte Gesundheitsberichterstattung allgemein [29] ein in Praxis und Politik nutzbares Instrument für die öffentliche Selbstdarstellung und die Mobilisierung zusätzlicher Ressourcen dar. Wünschenswert wäre jedoch eine darüber hinausgehende wissenschaftliche Legitimierung multimodaler Gesundheitsförderungsprogramme der lokalen Ebene. Diese Legitimierung hängt jedoch sehr stark von der wissenschaftlichen Qualität des Instruments und seiner allgemeinen Einsetzbarkeit, also seiner generischen Funktion, ab. Wie weit mithilfe des Instruments in allgemeiner Weise die Evidenz für die Wirksamkeit multimodaler Gesundheitsförderungsprogramme vergrößert werden kann, ist derzeit noch nicht zu sagen.

Wir glauben, dass Capacity Building, verstanden als nachhaltige Strukturbildung und Kompetenzentwicklung, im Prinzip als allgemeiner, intermediärer Erfolgsindikator gut geeignet ist. Die methodischen Probleme sind allerdings nicht unerheblich. Der Stellenwert des hier vorgestellten Messinstruments für Zwecke von einerseits Evaluationsforschung und andererseits kontinuierlicher Qualitätsentwicklung kann erst auf der Basis weiterer Erprobung und wissenschaftlicher Analyse beurteilt werden.

\section{Literatur}

1 Walter U, Schwartz FW. Prävention. In: Schwartz FW et al., Hrsg. Das Public Health Buch. Gesundheit und Gesundheitswesen. München und Jena: Urban \& Fischer; 2003; 189ff

2 Hawe P, King L, Noort M. Indicators to Help with Capacity Building in Health Promotion. Sidney: NSW Health; 2000

3 World Health Organization. Health Promotion Glossary. Geneva: WHO; 1998

4 Nutbeam D. Evaluating health promotion - progress, problems and solutions. Health Promotion International 1998; 13 (1): 27-44

5 Smith BJ, Tang KC, Nutbeam D. WHO Health Promotion Glossary: new terms. Health Promotion International 2006; 21 (4): 340-345

6 Labonte R. Empowerment: Notes on Professional and Community Dimensions. Canadian Review of Social Policy 1990; 26: 64-75

7 Maton KI, Salem DA. Organizational Characteristics of Empowering Community Settings: A Multiple Case Study Approach. American Journal of Community Psychology 1995; 23 (5): 631-657 
8 Goodman RM et al. Identifying and Defining the Dimensions of Community Capacity to Provide a Basis for Measurement. Health Educational \& Behavior 1998; 25 (3): 258-278

9 Jackson T, Mitchell S, Wright M. The Community Development Continuum. Community Health Studies 1989; XIII (1): 66-73

10 Minkler M, Wallerstein $N$. Improving Health through Community Organisation and Community Building. In: Glanz K et al. Health Behaviour and Health Education: Theory, Research and Practice. San Francisco: Jossey-Bass; 1997

11 Bjaras G, Haglund BJA, Rifkin S. A new Approach to Community Participation Assessment. Health Promotion International 1991; 6 (3): 199-206

12 Laverack G, Labonte R. A Planning Framework for Community Empowerment Goals within Health Promotion. Health Policy and Planning 2000; 15 (3): 255-262

13 Labonte R, Laverack G. Capacity Building in Health Promotion, Part 1: For whom? And for what Purpose? Critical Public Health 2001; 11 (2): $111-127$

14 Labonte R, Laverack G. Capacity Building in Health Promotion, Part 2: Whose Use? And with what Measurement? Critical Public Health 2001; 11 (2): 129-138

15 Gibbon $M$, Labonte R, Laverack G. Evaluating Community Capacity. Health and Social Care in the Community 2002; 10 (6): 485-491

16 Mossakowski $K$ et al. Quartiersdiagnose Lenzgesund - mehr Gesundheit ins Quartier! Daten und Ansätze zur Gesundheitsförderung in der Lenzsiedlung in Hamburg-Eimsbüttel. Hamburg: Eigenverlag; 2006

17 Mossakowski $K$ et al. Die Quartiersdiagnose: Daten und Ansätze für ein stadtteilorientiertes Präventionsprogramm des Öffentlichen Gesundheitsdienstes - erste Ergebnisse eines Forschungsprojektes. Prävention und Gesundheitsförderung 2007; 1 (2): 82-89

18 Kohler $S$ et al. Kindergesundheit in der Lenzsiedlung. Beiträge zur Quartiersdiagnose. Hamburg: Eigenverlag; 2007

19 Bush R, Dower J, Mutch A. Community Capacity Index, Version 2. Centre for Primary Health Care, University of Queensland; 2002
20 Kwan B et al. A Synthesis Paper on the Conceptualization and Measurement of Community Capacity. Institute of Health Promotion Research: UBC; 2003

21 Noack H, Reis-Klingspiegl K. Building Health Promotion Capacities in Local Settings: Community Readiness and Community Participation. In: Noack H, Kahr-Gottlieb D, Hrsg. Promoting The Public's Health. EUPHA 2005 Conference Book. Gamburg: G. Conrad; 2006; 113-122

22 Laverack G. Public Health: Power, Empowerment and Professional Practice. London: Palgrave Macmillan; 2005

23 Laverack G. Empowerment \& Health Promotion Programming: Part 2 Gesundheitswesen 2008: in diesem Heft

24 Hoeijmakers $M$, Leeuw E de, Kenis $P$ et al. Local Health policy development processes in the Netherlands: an expanded toolbox for health promotion. In: Health Pomotion International 2007; 22 (2): 112-121

25 Loss J, Eichhorn C, Gehlert J et al. Gemeindenahe Gesundheitsförderung - Herausforderung an die Evaluation. Gesundheitswesen 2007; 69: 77-87

26 Loss J, Eichhorn C, Reisig V et al. Qualitätsmanagement in der Gesundheitsförderung. Entwicklung eines multidimensionalen Qualitätssicherungsinstruments für eine landesweite Gesundheitsinitiative. Präv. Gesundheitsf. 2007; 2: 199-206

27 Kliche T, Töppisch J, Kawski S et al. Die Beurteilung der Struktur-, Konzept- und Prozessqualität von Prävention und Gesundheitsförderung: Anforderungen und Lösungen. Bundesgesundheitsblatt - Gesundheitsforschung - Gesundheitsschutz 2004; 47: 125-132

28 Stockmann R. Evaluation in Deutschland In: Stockmann R, Hrsg. Evaluationsforschung. Grundlagen und ausgewählte Forschungsfelder. Opladen: Leske \& Budrich Verlag; 2000

29 Süß W, Schäfer I, Trojan A, Hrsg. Integrierte (Gesundheits-) Berichte. Konzeptionelle Überlegungen und Umsetzungserfahrungen. Aachen: Shaker Verlag; 2007 